\title{
Minimally invasive liver resection in Japan: is the robot necessary?
}

\section{Takeaki Ishizawa, Kiyoshi Hasegawa}

Hepato-Biliary-Pancreatic Surgery Division, Department of Surgery, Graduate School of Medicine, The University of Tokyo, Tokyo 113-8655, Japan

Correspondence to: Kiyoshi Hasegawa, MD, PhD, FACS, Hepato-Biliary-Pancreatic Surgery Division, Department of Surgery, Graduate School of Medicine, The University of Tokyo, 7-3-1, Hongo, Bunkyo-ku, Tokyo 113-8655, Japan.

E-mail: hasegawa-2su@h.u-tokyo.ac.jp

How to cite this article: Ishizawa T, Hasegawa K. Minimally invasive liver resection in Japan: is the robot necessary? Miniinvasive Surg 2021;5:52. https://dx.doi.org/10.20517/2574-1225.2021.81

Received: 29 Jun 2021 First Decision: 20 Jul 2021 Revised: 17 Aug 2021 Accepted: 1 Sep 2021 Available online: 5 Nov 2021

Academic Editors: Andrew A. Gumbs, Kit Fai LEE Copy Editor: Xi-Jun Chen Production Editor: Xi-Jun Chen

\begin{abstract}
Robot-assisted hepatectomy (RAH) is rarely indicated in Japan because of the lack of reimbursement from the national health insurance system. Instead, laparoscopic hepatectomy has been approved for all hepatectomy procedures except resections requiring biliary reconstruction. An obvious advantage of RAH over laparoscopic hepatectomy is the fact that surgeons can use multi-articulated surgical devices, which may facilitate resection of superior/posterior hepatic regions, hilar dissection, biliary reconstruction, and hepatic segmentation by fluorescence imaging. With the accumulation of evidence supporting the use of robotic surgical devices in particular situations of hepatectomy, RAH will become more commonly indicated in Japan under the existing nationwide reporting system and board certification systems to assure surgical safety.
\end{abstract}

Keywords: Robot-assisted hepatectomy, laparoscopic hepatectomy, minimally invasive hepatectomy, anatomic hepatectomy, fluorescence imaging

\section{INTRODUCTION}

During the past three decades, the indications for laparoscopic hepatectomy (LH) have been dramatically extended based on technical, oncological, and regional factors. In Japan, LH for limited resections and left lateral sectionectomy was first reimbursed by the national health insurance system in 2010; this was 
5000

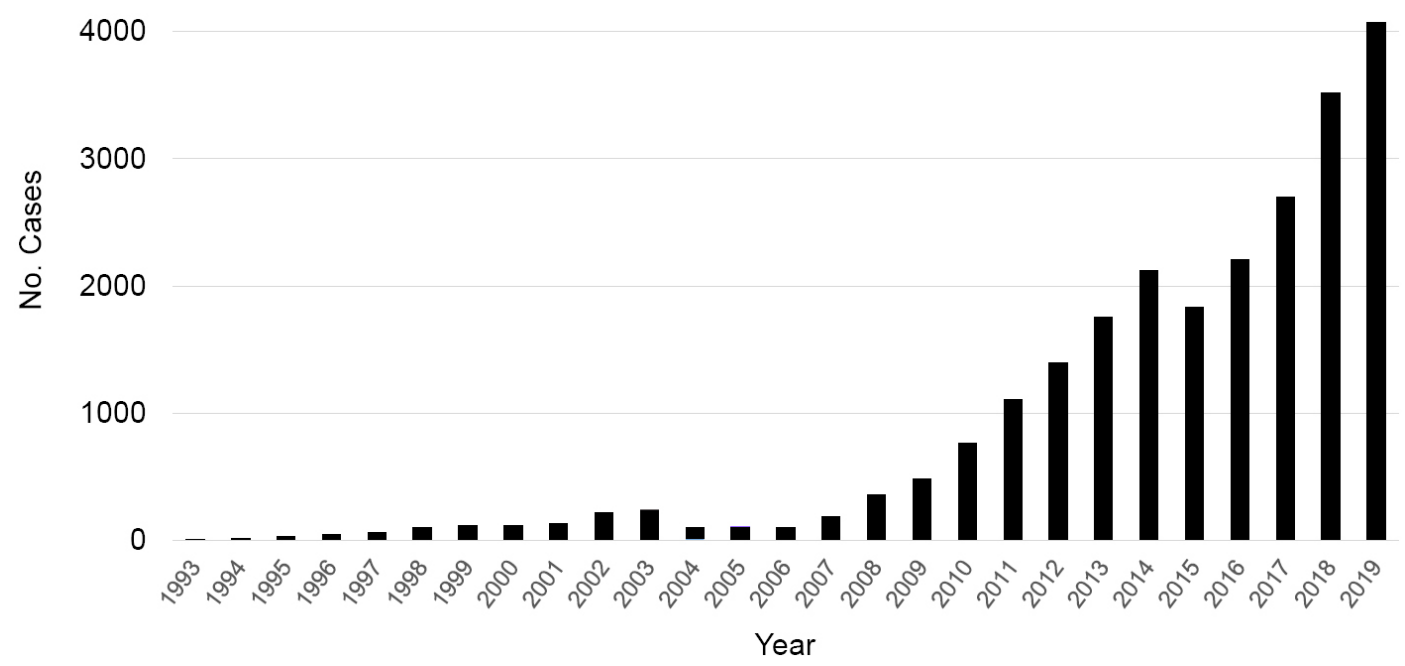

Figure 1. Trends of annual numbers of laparoscopic hepatectomies in Japan (1993-2019). Since the first insurance reimbursement in 2010, the numbers of laparoscopic hepatectomies have progressively increased, and this increase has been further boosted by the extension of insurance support to a wider range of hepatectomy procedures in 2016. This figure was created based on the 15th Nationwide Survey of Endoscopic Surgery.

followed by extension of its indications to all LH procedures except hepatectomy requiring biliary reconstruction in $2016^{[1]}$ and reimbursement of robot-assisted distal pancreatectomy and pancreaticoduodenectomy in 2020. As of 2021, however, robot-assisted hepatectomy (RAH) has not been reimbursed. We herein introduce the current status of minimally invasive hepatectomy in Japan and discuss the potential advantages of RAH over conventional hepatectomy procedures in an effort to promote future proliferation of robot-assisted hepatobiliary surgery.

\section{CURRENT DISSEMINATION STATUS OF RAH IN JAPAN}

Since the first insurance reimbursement in 2010, the number of LH cases in Japan has progressively increased, and this increase was further boosted by the extension of insurance support to a wider range of hepatectomy procedures in 2016 [Figure 1]. Based on the National Clinical Database, 13\% of hepatectomy procedures (more than one segment excluding the lateral segment) were performed laparoscopically in $2019^{[2]}$. In contrast, RAH has rarely been performed in Japan; according to the 15th Nationwide Survey of Endoscopic Surgery, RAH accounted for only $0.5 \%$ of all minimally invasive hepatectomy procedures performed in 459 medical centers belonging to the Japan Society for Endoscopic Surgery [Table 1, The 15th Nationwide Survey of Endoscopic Surgery in Japan (The Japanese Society for Endoscopic Surgery)]. In Japan, where all citizens receive medical care under the universal health insurance system, insurance reimbursement is critical for both patients and surgeons to ensure access to newly developed therapeutic modalities such as robot-assisted surgery.

Another feature to consider in the dissemination of RAH is the safety of hepatectomy in Japan. Even after the nationwide establishment of $\mathrm{LH}$, the overall mortality rates after hepatectomy remained quite low (0.7\%-1.4\% at 30 days and $1.3 \%-2.6 \%$ at 90 days from 2011 to 2019 ), with favorable morbidity rates (3.4\%$4.3 \%$ Clavien-Dindo grade IIIa-V complications $)^{[2]}$. Step-by-step establishment of LH under the board certification systems provided by the Japan Society for Endoscopic Surgery ${ }^{[3]}$, the Japanese Society of 
Table 1. Numbers of different minimally invasive hepatectomy procedures in Japan in $2019^{*}$

\begin{tabular}{lllc}
\hline & Pure LH & RAH & Others $^{\dagger}$ \\
\hline Wedge resection & 2663 & 9 & 131 \\
Couinaud's segmentectomy & 348 & 2 & 9 \\
Left lateral sectionectomy & 275 & 1 & 2 \\
Sectionectomy & 311 & 5 & 14 \\
Bisectionectomy & 260 & 0 & 17 \\
Trisectionectomy & 10 & $19(0.5 \%)$ & 1 \\
Total & $3867(94.9 \%)$ & $187(4.6 \%)$ & 15 \\
\hline
\end{tabular}

*Based on the 15th Nationwide Survey of Endoscopic Surgery in Japan (the Japan Society for Endoscopic Surgery). ${ }^{\dagger}$ Hand-assisted or hybrid procedures. LH: Laparoscopic hepatectomy; RAH: robot-assisted hepatectomy.

Hepato-Biliary-Pancreatic Surgery ${ }^{[4,5]}$, and the nationwide online registry system ${ }^{[6]}$ have contributed to the safe and consistent dissemination of LH in Japan.

\section{POSSIBLE ADVANTAGES OF PROMOTING FUTURE DISSEMINATION OF RAH}

Recent systemic reviews and meta-analyses have already shown that RAH offers acceptable operative outcomes at least comparable to those of $\mathrm{LH}$, except for possible extension of the operation time $\mathrm{e}^{[\mathrm{7}-9]}$. More recently, a meta-analysis focusing on major (three or more Couinaud's segments) hepatectomies suggested advantages of RAH over $\mathrm{LH}$ in decreasing a conversion rate and perioperative blood loss ${ }^{[10]}$. Considering the higher cost of $\mathrm{RAH}^{[7,9]}$, however, we need more evidence supporting the clear advantages of using robotic surgical systems in specific aspects of hepatectomy procedures, as suggested below, which enables selection of the patient appropriate to RAH.

\section{Resection of superior/posterior hepatic regions}

One of the major limitations of LH lies in the fact that conventional procedures allow only tangential movements of laparoscopic forceps. This makes deep wedge resections difficult to perform, especially for lesions located in the right superior/posterior regions of the liver. Using a "lateral approach" with intercostal trocars ${ }^{[1,1,2]}$ is a possible solution for $\mathrm{LH}$, but this technique may not be applicable to patients with a history of pulmonary disease or surgery. Vertical transection of the hepatic parenchyma enabled by multiarticulated movements of robotic devices may facilitate resection of hepatic tumors located in difficult regions, as suggested by a previous comparative study ${ }^{[13]}$. Melstrom et al.$^{[14]}$ also suggested efficacy of RAH in decreasing postoperative hospital stay (even on the day of surgery), especially in cases of superior/posterior hepatic regions where the incision for open surgery would dominate the course of recovery.

\section{Hilar dissection and biliary reconstruction}

Flexible movements of robotic surgical forceps also enable minute dissections of hepatic vessels running in the hilar plates and hepatoduodenal ligament; this may be associated with favorable operative outcomes with a lower probability of open conversion in major hepatectomies and complicated hepatectomy procedures requiring hilar dissection as compared with $\mathrm{LH}^{[7,10,15]}$. Suturing with the use of multi-articulated needle holders is an obvious advantage of robot-assisted surgery over conventional laparoscopic techniques. In the context of hepatobiliary surgery, this feature would work most effectively for biliary anastomosis as demonstrated in surgery for choledochal $\mathrm{cysts}^{[16]}$, although no $\mathrm{LH}$ procedures requiring biliary reconstruction have been reimbursed by the Japanese health insurance system to date. 


\section{Hepatic segmentation by fluorescence imaging for anatomic resection}

Because the latest robotic surgical systems are equipped with near-infrared imaging technology (da Vinci Firefly; Intuitive Surgical, Sunnyvale, CA, USA), intraoperative fluorescence imaging using indocyanine green (ICG) can be easily applied to RAH as well as LH and open hepatectomy for real-time visualization of the biliary anatomy (fluorescence cholangiography), liver cancers, and boundaries of hepatic segments ${ }^{[17]}$. Among these procedures, hepatic segmentation can be achieved by direct injection of ICG into the target portal branch (positive staining technique) ${ }^{[18,19]}$ or by systemic injection of ICG following closure of the portal pedicle feeding tumor-bearing hepatic segments (negative staining technique ${ }^{[1,20]}$. If a robotic surgical system could be used to perform a positive staining technique, it would facilitate easier puncture of the target portal branch under ultrasound guidance compared with laparoscopic needle manipulation ${ }^{[21]}$. The use of robotic surgical devices also enables multidirectional dissection of the hepatic hilum to reach the corresponding Glissonian sheaths to be divided ${ }^{[22]}$, which may extend the indications for the negative staining technique to anatomic resection of deeply located hepatic segments. Although near-infrared imaging has been installed in the latest model of laparoscopic imaging systems as well as robotic surgical systems, use of this technology with multi-articulated forceps and three-dimensional color imaging may further extend applications of fluorescence imaging during hepatobiliary surgery.

\section{Integrated surgical navigation, autonomous actions, and surgical decision-making by artificial intelligence}

In addition to intraoperative information obtained by techniques such as fluorescence imaging and ultrasonography, preoperative simulation can be placed in the surgeon's console of the robotic surgical system and displayed in real time with three-dimensional images of operative fields. In this respect, RAH has a potential advantage over LH in terms of the ability of surgeons to understand special relationships between anatomical structures and tumors by integrating preoperative and intraoperative imaging information. Applications of augmented reality ${ }^{[23]}$ and artificial intelligence ${ }^{[24]}$ may further promote the development of surgical navigation systems. In addition, application of artificial intelligence in robotic surgery may enable autonomous control of surgical installments like a laparoscope and staplers and provide precision information for accurate surgical decision-making ${ }^{[25]}$.

\section{POSSIBLE DISADVANTAGES OF APPLYING ROBOT-ASSISTED SURGERY TO HEPATECTOMY}

As mentioned previously, the major disadvantage of RAH over LH is the higher cost associated with the initial installation and use of each instalment. Especially in Japan, the amount of future insurance claim for RAH may be the same as that for LH as in the case of pancreatectomy, which can press management of the medical institutions. Limited lineup of aspiration and dissection devices (no angular ultrasonic dissectors) designed for hepatic parenchymal transection is another drawback of RAH, leading to longer operation time than LH as demonstrated in previous studies ${ }^{[7-9]}$. We expect that the next-generation robotic surgical systems are devised with the opinions from liver surgeons to adjust to the specific conditions of hepatectomy.

\section{CONCLUSIONS}

With the accumulation of evidence indicating the specific advantages of RAH over LH, robotic surgical systems will become more commonly used for hepatobiliary surgery in Japan as well as in other countries. After reimbursement by the health insurance system, we aim to apply RAH with prioritization of surgical safety using a nationwide reporting system and board certification systems for the performance of LH and robot-assisted pancreatic resections. 


\section{DECLARATIONS}

\section{Acknowledgments}

We thank Angela Morben, DVM, ELS, from Edanz (https://jp.edanz.com/ac) for editing a draft of this manuscript.

\section{Authors' contributions}

Made substantial contributions to the conception and design of the study, performed the data analysis and interpretation, and wrote the manuscript: Ishizawa $\mathrm{T}$

Provided administrative and technical support and critical revisions of the manuscript: Hasegawa K

\section{Availability of data and materials}

Not applicable.

\section{Financial support and sponsorship}

None.

\section{Conflicts of interest}

Both authors declared that there are no conflicts of interest.

\section{Ethical approval and consent to participate}

Not applicable.

\section{Consent for publication}

Not applicable.

\section{Copyright}

(c) The Author(s) 2021.

\section{REFERENCES}

1. Kaneko H, Otsuka Y, Kubota Y, Wakabayashi G. Evolution and revolution of laparoscopic liver resection in Japan. Ann Gastroenterol Surg 2017;1:33-43. DOI PubMed PMC

2. Marubashi S, Takahashi A, Kakeji Y, et al; the National Clinical Database. Surgical outcomes in gastroenterological surgery in Japan: Report of the National Clinical Database 2011-2019. Ann Gastroenterol Surg 2021. DOI

3. Mori T, Kimura T, Kitajima M. Skill accreditation system for laparoscopic gastroenterologic surgeons in Japan. Minim Invasive Ther Allied Technol 2010;19:18-23. DOI PubMed

4. Miura F, Yamamoto M, Gotoh M, et al. Validation of the board certification system for expert surgeons (hepato-biliary-pancreatic field) using the data of the National Clinical Database of Japan: part 1 - Hepatectomy of more than one segment. J Hepatobiliary Pancreat Sci 2016;23:313-23. DOI PubMed

5. Arita J, Yamamoto H, Kokudo T, et al. Impact of board certification system and adherence to the clinical practice guidelines for liver cancer on post-hepatectomy risk-adjusted mortality rate in Japan: a questionnaire survey of departments registered with the National Clinical Database. J Hepatobiliary Pancreat Sci 2021. DOI PubMed

6. Wakabayashi G, Kaneko H. Can major laparoscopic liver and pancreas surgery become standard practices? J Hepatobiliary Pancreat Sci 2016;23:89-91. DOI PubMed

7. Zhang L, Yuan Q, Xu Y, Wang W. Comparative clinical outcomes of robot-assisted liver resection versus laparoscopic liver resection: a meta-analysis. PLoS One 2020;15:e0240593. DOI PubMed PMC

8. Ziogas IA, Giannis D, Esagian SM, Economopoulos KP, Tohme S, Geller DA. Laparoscopic versus robotic major hepatectomy: a systematic review and meta-analysis. Surg Endosc 2021;35:524-35. DOI PubMed

9. Wang JM, Li JF, Yuan GD, He SQ. Robot-assisted versus laparoscopic minor hepatectomy: A systematic review and meta-analysis. Medicine (Baltimore) 2021;100:e25648. DOI PubMed PMC

10. Coletta D, Levi Sandri GB, Giuliani G, Guerra F. Robot-assisted versus conventional laparoscopic major hepatectomies: systematic review with meta-analysis. Int J Med Robot 2021;17:e2218. DOI PubMed

11. Ishizawa T, Gumbs AA, Kokudo N, Gayet B. Laparoscopic segmentectomy of the liver: from segment I to VIII. Ann Surg 2012;256:959-64. DOI PubMed

12. Ichida H, Ishizawa T, Tanaka M, et al. Use of intercostal trocars for laparoscopic resection of subphrenic hepatic tumors. Surg Endosc 2017;31:1280-6. DOI PubMed 
13. Chong CCN, Lok HT, Fung AKY, et al. Robotic versus laparoscopic hepatectomy: application of the difficulty scoring system. Surg Endosc 2020;34:2000-6. DOI PubMed

14. Melstrom LG, Warner SG, Woo Y, et al. Selecting incision-dominant cases for robotic liver resection: towards outpatient hepatectomy with rapid recovery. Hepatobiliary Surg Nutr 2018;7:77-84. DOI PubMed PMC

15. Fruscione M, Pickens R, Baker EH, et al. Robotic-assisted versus laparoscopic major liver resection: analysis of outcomes from a single center. HPB (Oxford) 2019;21:906-11. DOI PubMed

16. Chi SQ, Cao GQ, Li S, et al. Outcomes in robotic versus laparoscopic-assisted choledochal cyst excision and hepaticojejunostomy in children. Surg Endosc 2021;35:5009-14. DOI PubMed

17. Wang X, Teh CSC, Ishizawa T, et al. Consensus guidelines for the use of fluorescence imaging in hepatobiliary surgery. Ann Surg 2021;274:97-106. DOI PubMed

18. Ishizawa T, Zuker NB, Kokudo N, Gayet B. Positive and negative staining of hepatic segments by use of fluorescent imaging techniques during laparoscopic hepatectomy. Arch Surg 2012;147:393-4. DOI PubMed

19. Ito D, Ishizawa $\mathrm{T}$, Hasegawa $\mathrm{K}$. Laparoscopic positive staining of hepatic segments using indocyanine green-fluorescence imaging. $J$ Hepatobiliary Pancreat Sci 2020;27:441-3. DOI PubMed

20. Terasawa M, Ishizawa T, Mise Y, et al. Applications of fusion-fluorescence imaging using indocyanine green in laparoscopic hepatectomy. Surg Endosc 2017;31:5111-8. DOI PubMed

21. Chiow AKH, Rho SY, Wee IJY, Lee LS, Choi GH. Robotic ICG guided anatomical liver resection in a multi-centre cohort: an evolution from "positive staining" into "negative staining" method. HPB (Oxford) 2021;23:475-82. DOI PubMed

22. Berardi G, Igarashi K, Li CJ, et al. Parenchymal sparing anatomical liver resections with full laparoscopic approach: description of technique and short-term results. Ann Surg 2021;273:785-91. DOI PubMed

23. Pessaux P, Diana M, Soler L, Piardi T, Mutter D, Marescaux J. Towards cybernetic surgery: robotic and augmented reality-assisted liver segmentectomy. Langenbecks Arch Surg 2015;400:381-5. DOI PubMed

24. Panesar S, Cagle Y, Chander D, Morey J, Fernandez-Miranda J, Kliot M. Artificial intelligence and the future of surgical robotics. Ann Surg 2019;270:223-6. DOI PubMed

25. Gumbs AA, Perretta S, d'Allemagne B, Chouillard E. What is artificial intelligence surgery? Art Int Surg 2021;1:1-10. DOI 